\title{
The Value of Prognostic Nutritional Index (PNI) on Newly Diagnosed Diffuse Large B-Cell Lymphoma Patients: A Multicenter Retrospective Study of HHLWG Based on Propensity Score Matched Analysis
}

Ziyuan Shen, '* Fei Wang, ${ }^{2, *}$ Chenlu He, ${ }^{1} *$ Dashan $\mathrm{Li}^{3}{ }^{3}$ Shanlin Nie, ${ }^{3}$ Zhenzhen Bian, (D) ${ }^{3}$ Mingkang Yao, Yuhao Xue, ${ }^{5}$ Ying Wang, ${ }^{6}$ Weiying Gu, ${ }^{2}$ Taigang Zhu, ${ }^{7}$ Yuye Shi, ${ }^{5}$ Hao Zhang, Shuiping Huang, ${ }^{1,8}$ Yuqing Miao,

Wei Sang ${ }^{3}$

On behalf of the Huaihai Lymphoma

Working Group

'Department of Epidemiology and Biostatistics, Xuzhou Medical University, Xuzhou, Jiangsu, People's Republic of China; ${ }^{2}$ Department of Hematology, The First People's Hospital of Changzhou, Changzhou, Jiangsu, People's Republic of China; ${ }^{3}$ Department of Hematology, Affiliated Hospital of Xuzhou Medical University, Xuzhou, Jiangsu, People's Republic of China; ${ }^{4}$ Department of Hematology, Affiliated Hospital of Jining Medical University, Jining, Shandong, People's Republic of China; ${ }^{5}$ Department of Hematology, The First People's Hospital of Huaian, Huaian, Jiangsu, People's Republic of China; ${ }^{6}$ Department of Personnel, Suqian First Hospital, Suqian, Jiangsu, People's Republic of China; 'Department of Hematology, The General Hospital of Wanbei Coal-Electric Group, Suzhou, People's Republic of China; ${ }^{8}$ Center for Medical Statistics and Data Analysis, Xuzhou Medical University, Xuzhou, Jiangsu, People's Republic of China; 'Department of Hematology, Yancheng First People's Hospital, Yancheng, Jiangsu, People's Republic of China

*These authors contributed equally to this work

Correspondence: Wei Sang

Department of Hematology, Affiliated Hospital of Xuzhou Medical University, Xuzhou, Jiangsu, People's Republic of China

Tel +8613645207648

Email xyfylbl515@xzhmu.edu.cn

Yuqing Miao

Department of Hematology, Yancheng First

People's Hospital, Yancheng, Jiangsu, People's

Republic of China

Tel +8613851342032

Email miaomiaomyq@I63.com
Introduction: Immunonutritional status is associated with the survival of DLBCL. This multicenter retrospective study aimed to explore the prognostic value of Prognostic Nutrition Index (PNI) in DLBCL patients by using propensity score matched analysis (PSM).

Methods: A total of 990 DLBCL cases were recruited from 5 centers of Huaihai Lymphoma Working Group (HHLWG). A 1:1 PSM analysis was performed using the nearest-neighbor method, with a caliper size of 0.02 . Cox regression analysis was used to examine factors associated with survival.

Results: The median age at diagnosis was 62 years and $52.5 \%$ were males, with the 3 -y overall survival of $65.1 \%$. According to the MaxStat analysis, 44 was the optimal cutoff point of PNI. After PSM analysis, a total of 282 patients in PNI $<44$ group could be propensity matched to $\mathrm{PNI} \geq 44$ patients, creating a group of 564 patients. Multivariable analysis revealed that PNI, age, central nervous system involvement and International Prognostic Index (IPI) were independent prognostic factors for DLBCL. Kaplan-Meier analysis indicated that patients with low PNI in Ann Arbor Stage (III/VI), ECOG (<2), IPI (LR+LIR), GCB, and BCL-2 negative groups had a poor prognosis.

Discussion: PNI could accurately stratify the prognosis of DLBCL after PSM analysis.

Keywords: diffuse large B-cell lymphoma, prognostic nutrition index, propensity score matched, prognosis

\section{Introduction}

Diffuse large B-cell lymphoma (DLBCL) is the most common subtype of nonHodgkin's lymphoma with high heterogeneity and aggressiveness. Although $60-70 \%$ patients can be cured by the current standard of care in the frontline setting, the majority of the remaining patients will experience refractory and relapse. Clinical variables based prognostic systems, such as International Prognostic Index (IPI) ${ }^{1}$ and NCCN$\mathrm{IPI}^{2}{ }^{2}$ can achieve prognostic stratification of DLBCL patients. However, these indices do not take the nutritional status of patients into account.

Malnutrition is a common problem in cancer patients, occurring in up to $80 \%$ of patients with advanced stage. ${ }^{3}$ It will affect the response to treatment and lead to poor quality of life in cancer patients. ${ }^{4}$ Assessment of systemic nutritional status was 
refined by the introduction of the prognostic nutrition index (PNI), a continuous variable based on serum albumin concentration and total lymphocyte count in peripheral blood. ${ }^{5}$ PNI was originally designed to assess perioperative immunonutritional status and surgical risk in patients undergoing gastrointestinal surgery. ${ }^{6,7}$ Several studies have confirmed that PNI has prognostic value in a variety of malignant tumors. $^{6,8,9}$ Yao et al suggested that PNI was a significant indicator of extranodal natural killer/T cell lymphoma. ${ }^{10}$ Besides, there were a few reports exploring the prognostic role of PNI in DLBCL with limitations of small sample size and single-center data. ${ }^{11-13}$

Confounding factors always weaken the accuracy and objectivity of retrospective studies. Propensity score analysis (PSM) is commonly used to overcome selection bias, adjust the confounding factors, improve the comparability between groups by increasing the evidence level in retrospective studies, and mimic randomization on observed covariates. ${ }^{14-18}$

In this retrospective study, based on multicenter data of DLBCL from Huaihai Lymphoma Working Group (HHLWG) in China, we firstly carried out this PSM analysis to evaluate the prognostic value of PNI in DLBCL patients.

\section{Materials and Methods}

\section{Patients}

A total of 990 newly diagnosed DLBCL patients from 2008 to 2021 were recruited, and all pathological biopsies were double blinded and reviewed by at least two pathologists. Exclusion criteria: 1) patients with other malignant tumors; 2) patients with special types of lymphoma (primary central nervous system lymphoma, primary mediastinal DLBCL, transformed DLBCL); 3) patients with other immune diseases; 4) diseases that affect the lymphocyte level and albumin level of patients (such as hepatitis, cirrhosis, chronic kidney disease). During admission, the following variables were considered: age, gender, lactate dehydrogenase (LDH), hemoglobin (HB), platelet (PLT), albumin (Alb), lymphocyte count (LYC), red blood cell count (RBC), Ki-67, presence of bulky disease, B symptoms, $\beta_{2}$-microglobulin $\left(\beta_{2}-\mathrm{MG}\right)$, ECOG PS and Ann Arbor stage.

\section{Follow-Up and Endpoints}

Follow-up was conducted by consulting inpatient medical records and making phone calls. We followed up all the patients until July 28, 2021 or the death of patients. Overall survival (OS) was calculated as the interval between the time of diagnosis and death from any cause or the last follow-up. The survival status of all patients was confirmed with death records or a telephone call to next of kin (if patient died during the follow-up) or to the patients themselves. Study approval was obtained from the independent Ethics Committees of each participating center in HHLWG and met with the Helsinki Declaration.

\section{Assessment of PNI}

PNI, based on serum albumin and lymphocytes, is a scoring system that reflects the nutritional status and immune status of patients. It is calculated using the formula: ${ }^{7}$

$\mathrm{PNI}=10 \times \operatorname{albumin}(\mathrm{g} / \mathrm{dL})+0.005 \times$ lymphocyte count $\left(/ \mathrm{mm}^{3}\right)$

\section{Statistical Analysis}

Data were presented as numbers (percentages) for categorical variables and median (interquartile range, IQR) for all continuous variables. Differences in clinical factors were analyzed by using $\chi^{2}$ test. PNI was transformed into a categorical variable by MaxStat analysis (titled as Maximally Selected Rank Statistics). The Cox proportional hazard model was used to analyze the univariate association between prognostic factors and OS. All variables with $P<$ 0.05 in univariable analysis were kept in the multivariate analysis by using backward selection for the best predictor set, and Akaike Information Criteria (AIC) was used to evaluate the model. All the statistical tests were two-sided, and the statistical significance was set at $P<0.05$.

A 1:1 propensity score-matched (PSM) analysis was performed using the nearest-neighbor method, with a caliper size of 0.02 to identify the impact of PNI on DLBCL. To reduce selection bias and confounding factors, the propensity score was calculated using logistic regression analysis. The standardized mean difference (SMD) was measured to determine the balance between the two groups before and after PSM. The SMD cut-off value was $<0.1$, which was regarded as indicating a sufficient balance between the two groups. After 1:1 propensity score matching, continuous variables were compared using MannWhitney $U$-test, and categorical variables were compared using $\chi^{2}$ test. All statistical analyses were performed by SPSS statistics for Windows, Version 22.0 (Armonk, NY: IBM corp.), R software (version 4.0.3; http://www. Rproject.org) and Stata version 15.0. 


\section{Results}

\section{Characteristics of DLBCL Patients}

The characteristics of 990 DLBCL patients were detailed in Table 1. The median follow-up time was 46.133 (95\% CI: 43.759-48.508) months and the median OS was
140.033 (95\% CI: 70.614-209.453) months. At the end of follow-up, a total of 358 (36.1\%) deaths occurred. The median age at diagnosis was 62 years (range: 10-91), with $520(52.5 \%)$ males and $431(43.5 \%)$ patients were younger than 60 years. A total of $261(26.3 \%)$ patients with

Table I Associations Between PNI and Clinicopathological Factors Before and After PSM

\begin{tabular}{|c|c|c|c|c|c|c|}
\hline \multirow[t]{3}{*}{ Variables } & \multicolumn{2}{|c|}{ Before Propensity Matching } & \multirow[t]{3}{*}{$P$} & \multicolumn{2}{|c|}{ After Propensity Matching } & \multirow[t]{3}{*}{$P$} \\
\hline & PNI $<44$ & PNI $\geq 44$ & & PNI $<44$ & PNI $\geq 44$ & \\
\hline & $(n=318)$ & $(n=672)$ & & $(n=282)$ & $(n=282)$ & \\
\hline Age $(y)$ & $64(56,72)$ & $6 I(5 I, 68)$ & $<0.001$ & $63(54,7 I)$ & $66(57,71)$ & 0.093 \\
\hline $\begin{array}{l}\text { Gender } \\
\text { Male } \\
\text { Female }\end{array}$ & $\begin{array}{l}\text { I } 76(55.3) \\
\text { I } 42(44.7)\end{array}$ & $\begin{array}{l}344(5 \mathrm{I} .2) \\
328(48.8)\end{array}$ & 0.221 & $\begin{array}{l}152(53.9) \\
\text { I } 30(46.1)\end{array}$ & $\begin{array}{l}\text { I } 68 \text { (59.6) } \\
\text { II4 (40.4) }\end{array}$ & 0.174 \\
\hline PLT & $198(139,281)$ & $223(|83,27|)$ & $<0.001$ & $203(140,282)$ & $220(|65,26|)$ & 0.477 \\
\hline$\beta 2-M G$ & $\begin{array}{c}2.7 I \\
(2.63,5.1 I)\end{array}$ & $\begin{array}{c}2.71 \\
(2.05,4.48)\end{array}$ & 0.001 & $2.7(2.5,4.5)$ & $2.7(2.2,1710)$ & 0.433 \\
\hline NE & $\begin{array}{c}4.13 \\
(2.78,6.16)\end{array}$ & $\begin{array}{c}3.99 \\
(3.04,4.98)\end{array}$ & 0.315 & 4.I $(2.8,6.1)$ & $3.9(3.1-5.0)$ & 0.814 \\
\hline $\begin{array}{l}\text { LDH } \\
\text { Normal } \\
\text { Elevated }\end{array}$ & $\begin{array}{l}121(38.1) \\
197(61.9)\end{array}$ & $\begin{array}{l}454(67.6) \\
218(32.4)\end{array}$ & $<0.001$ & $\begin{array}{l}\text { II } 3(40.1) \\
\text { I } 69(59.9)\end{array}$ & $\begin{array}{l}\text { I } 58(56.0) \\
\text { I } 24(44.0)\end{array}$ & 0.107 \\
\hline $\begin{array}{l}\text { CNS involvement } \\
\text { Absence } \\
\text { Presence }\end{array}$ & $\begin{array}{c}249(78.3) \\
69(21.7)\end{array}$ & $\begin{array}{l}557(82.9) \\
115(17.1)\end{array}$ & 0.083 & $\begin{array}{c}227(80.5) \\
55(19.5)\end{array}$ & $\begin{array}{c}216(76.6) \\
66(23.4)\end{array}$ & 0.259 \\
\hline $\begin{array}{l}\text { BM involvement } \\
\text { Absence } \\
\text { Presence }\end{array}$ & $\begin{array}{c}246(77.4) \\
72(22.6)\end{array}$ & $\begin{array}{c}589(87.6) \\
83(12.4)\end{array}$ & 0.001 & $\begin{array}{c}225(79.8) \\
57(20.2)\end{array}$ & $\begin{array}{c}223(79.1) \\
59(20.9)\end{array}$ & 0.835 \\
\hline $\begin{array}{l}\text { Bulky } \\
\text { Absence } \\
\text { Presence }\end{array}$ & $\begin{array}{l}\text { I8I (56.9) } \\
\text { I37 (43.I) }\end{array}$ & $\begin{array}{l}548(8 \mid .5) \\
124(I 8.5)\end{array}$ & $<0.001$ & $\begin{array}{l}\text { I } 67(59.3) \\
\text { II }(40.7)\end{array}$ & $\begin{array}{c}209(74.1) \\
73(25.9)\end{array}$ & 0.148 \\
\hline $\begin{array}{c}\text { ECOG } \\
<2 \\
2-4\end{array}$ & $\begin{array}{l}192(60.4) \\
\text { I26 (39.6) }\end{array}$ & $\begin{array}{l}532(79.2) \\
140(20.8)\end{array}$ & $<0.001$ & $\begin{array}{c}184(65.2) \\
98(34.8)\end{array}$ & $\begin{array}{l}175(62.1) \\
107(37.9)\end{array}$ & 0.431 \\
\hline $\begin{array}{l}\text { IPI } \\
\qquad \begin{array}{l}\text { LR/+LIR } \\
\text { HIR/HR }\end{array}\end{array}$ & $\begin{array}{l}\text { I30 (40.9) } \\
\text { I88 (59.I) }\end{array}$ & $\begin{array}{l}495(73.7) \\
I 77(26.3)\end{array}$ & $<0.001$ & $\begin{array}{l}\text { I } 30(46.1) \\
\text { I52 (53.9) }\end{array}$ & $\begin{array}{l}\text { I27 (45.0) } \\
\text { I55 (55.0) }\end{array}$ & 0.800 \\
\hline $\begin{array}{l}\text { Ann Arbor stage } \\
\text { I/II } \\
\text { III/IV }\end{array}$ & $\begin{array}{c}94(29.6) \\
224(70.4)\end{array}$ & $\begin{array}{l}378(56.3) \\
294(43.8)\end{array}$ & $<0.001$ & $\begin{array}{c}92(32.6) \\
190(67.4)\end{array}$ & $\begin{array}{c}83(29.4) \\
199(70.6)\end{array}$ & 0.413 \\
\hline $\begin{array}{l}\text { Cell of origin } \\
\qquad \text { GCB } \\
\text { Non-GCB }\end{array}$ & $\begin{array}{l}\text { I } 75(55.0) \\
\text { I43 (45.0) }\end{array}$ & $\begin{array}{l}402(59.8) \\
270(40.2)\end{array}$ & 0.154 & $\begin{array}{l}156(55.3) \\
126(44.7)\end{array}$ & $\begin{array}{l}149(52.8) \\
133(47.2)\end{array}$ & 0.554 \\
\hline
\end{tabular}

Abbreviations: PLT, platelet; NE, neutrophil count; LDH, lactate dehydrogenase (normal<240 U/L); CNS involvement, central nervous system involvement; BM involvement, bone marrow involvement. 
B symptoms and 117 (11.8\%) patients were with bulky disease. The 5-y of patients were $65.1 \%$ (Figure 1). In this study, patients received regimens of CHOP-like $(n=261)$, R-CHOP/R-CHOP-like $(\mathrm{n}=601)$ and R-based intensive regimens $(n=128)$. Twenty-five patients received BTK inhibitor (BTKi), forty-four patients received methotrexate (MTX), and thirty-five patients received autologous hematopoietic stem cell transplantation (Auto-HSCT). KaplanMeier analysis indicated that there was no significant difference in therapeutic regimens in global comparison $(P>0.05)$. Further subgroup analysis of R-based intensive regimens also showed no difference.

\section{The Optimal Cut-off Point for PNI Based on MaxStat}

According to the maximal chi-square method, 44 was the optimal cut-off point that distinguished between two prognostic groups most effectively $\left(P<0.05, \chi^{2}=7.28\right.$, Figure 2$)$.
Applying this result, the new definition of $\mathrm{PNI}<44$ was poor nutritional status group, and PNI $\geq 44$ was normal nutritional status group.

\section{Characteristics of DLBCL Patients After PSM}

In this study, the Pseudo $\mathrm{R}^{2}$ before matching was about 0.09 , which decreased to less than 0.01 after matching, indicating that the overall equilibrium test could pass. After PSM, a total of 282 patients in PNI $<44$ group could be propensity matched to PNI $\geq 44$ patients, creating a group of 564 patients. The two groups were remarkably balanced after matching based on 8 confounding factors, as each confounding factor was no longer be significantly different $(P>0.05$, Table 1). The absolute standard deviation of the two matched groups were $<10 \%$ for all the covariates (Figure 3).

After PSM, the median OS of DLBCL patients was 34 months (95\% CI: 15.042-52.824) in low PNI group and 61 months (95\% CI: 31.795-90.205) in high PNI group. Ages

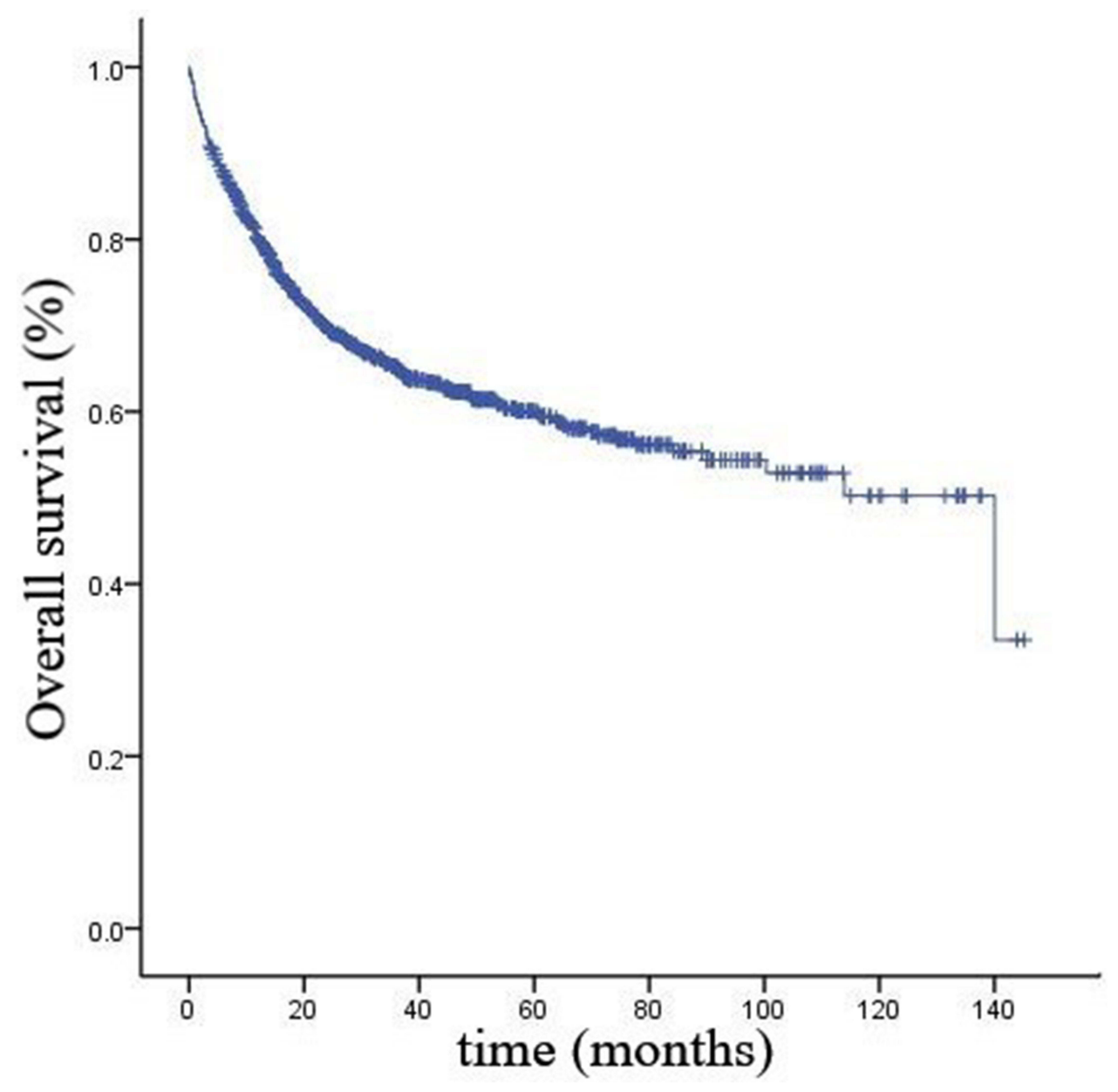

Figure I Overall survival of $990 \mathrm{DLBCL}$ patients. 


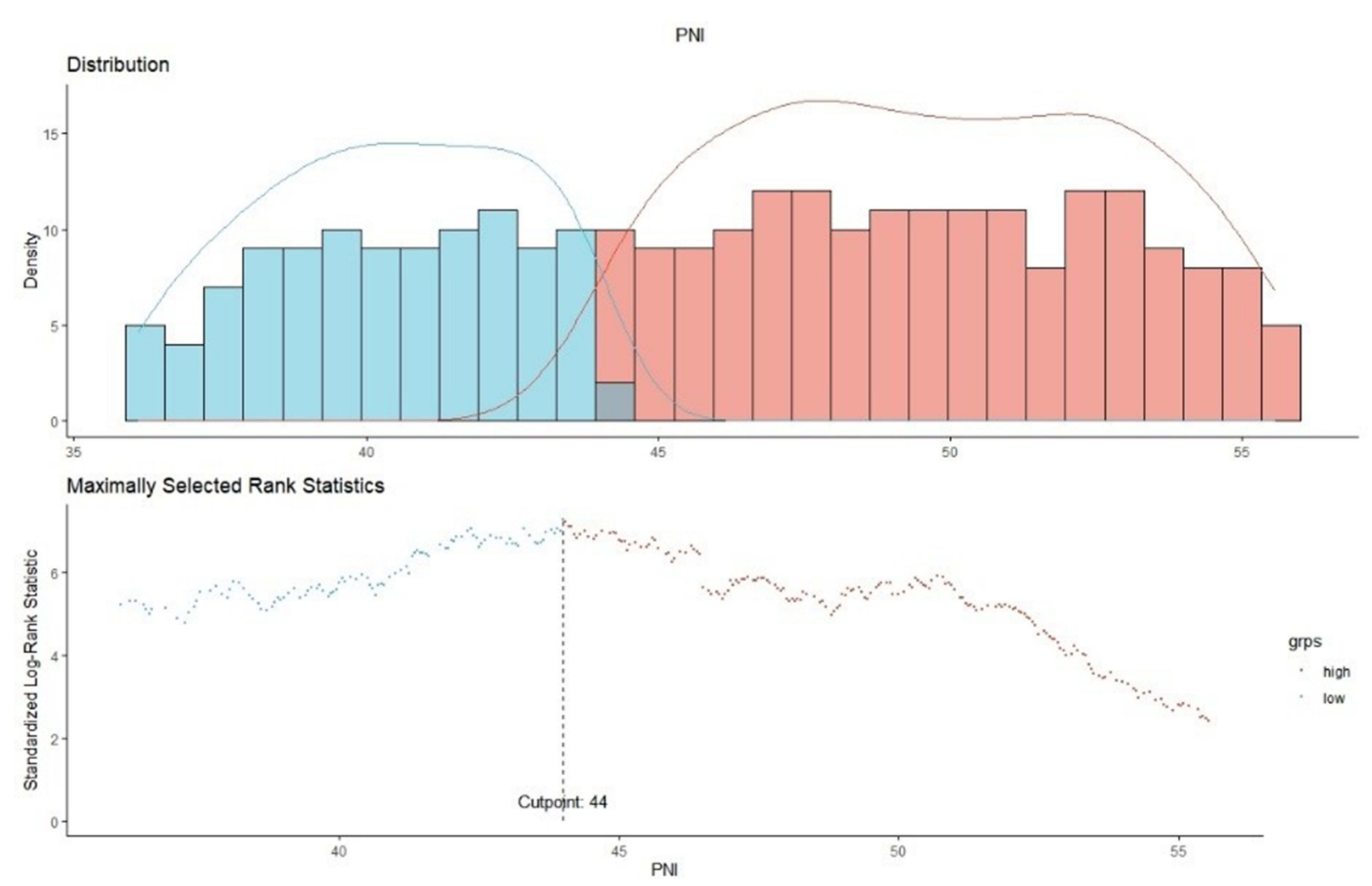

Figure 2 The optimal cut-off point for PNI by using MaxStat.

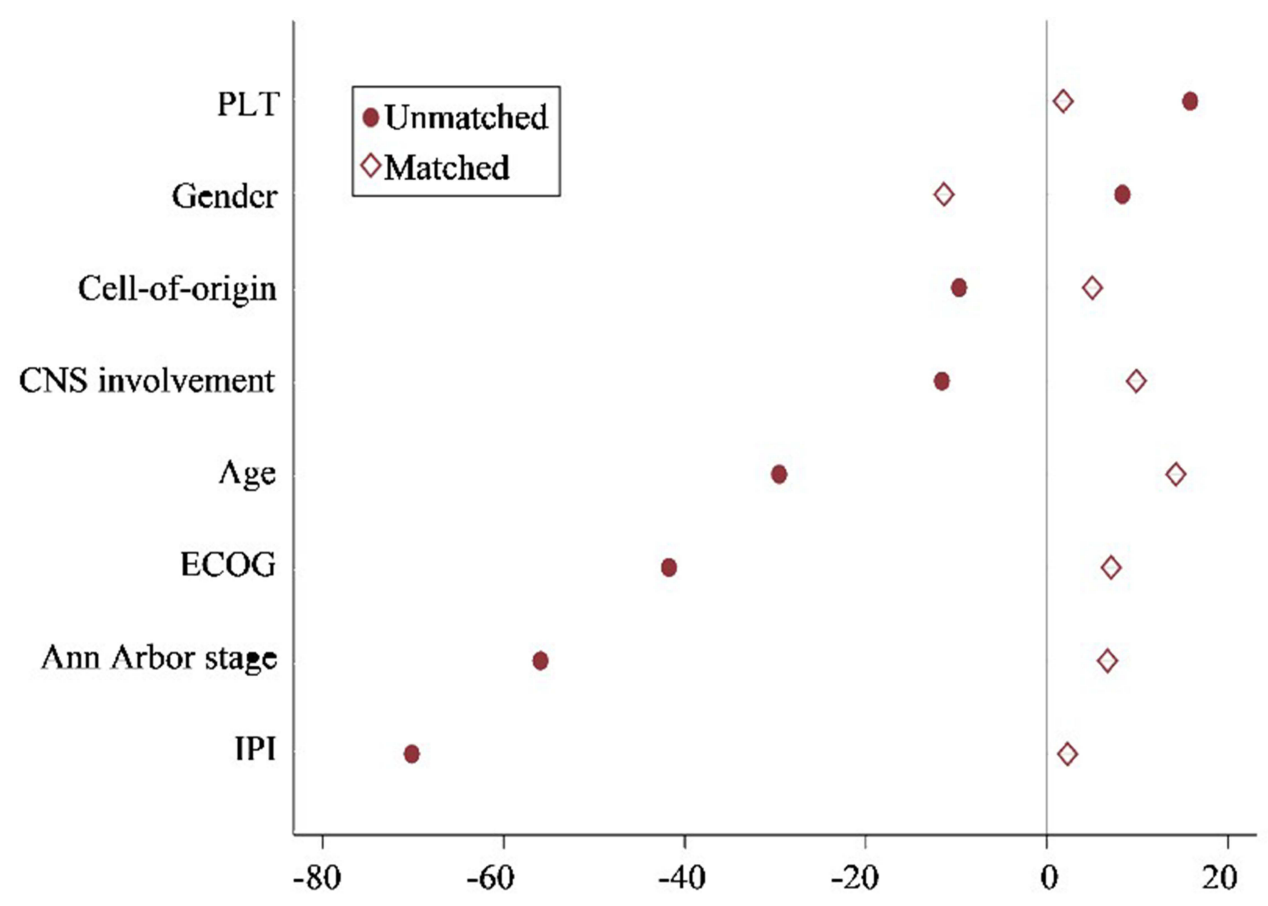

Absolute Standardized Difference \%

Figure 3 Impact of PNI on DLBCL after PSM. 
Hazard ratio ( $95 \%$ confidence interval)

(PNI $<44$ group vs. PNI $\geq 44$ group)

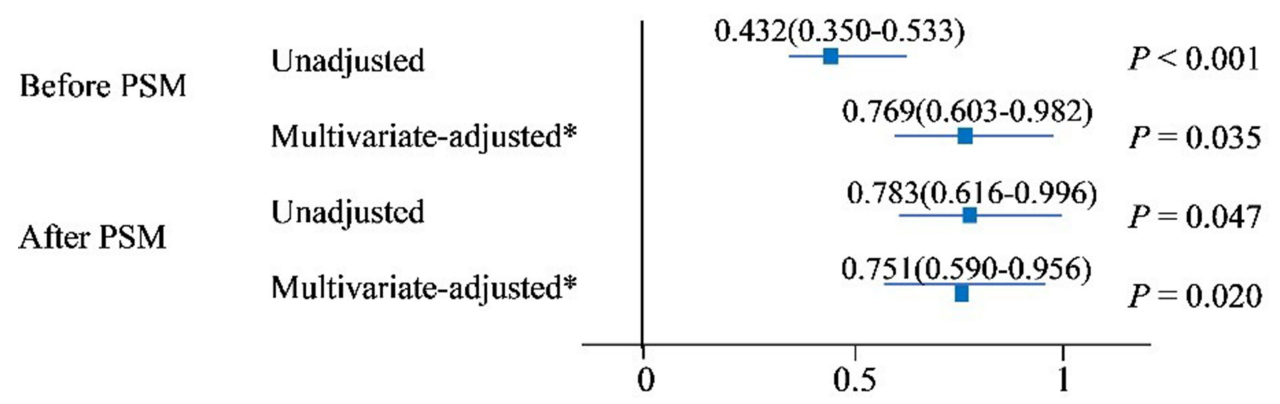

Figure 4 Predictive value of the PNI for the prognosis of DLBCL $(* P<0.05)$.

of patients ranged from 14 to 91 years with a median age of 65 years, of whom $244(43.2 \%)$ patients were female. A total of $121(27.1 \%)$ patients $(21.4 \%)$ patients were with CNS involvement and $87(15.4 \%)$ patients were with bulky disease. The characteristics of patients after PSM were detailed in Table 1.

The predictive value of the PNI $<44$ group for the prognosis of DLBCL was shown in Figure 4. Compared with $\mathrm{PNI} \geq 44$, PNI $<44$ was associated with poorer survival of DLBCL in unadjusted $(\mathrm{HR}=0.432,95 \% \mathrm{CI}$ : $0.350-0.533$; $P<0.001)$, multivariate-adjusted ( $\mathrm{HR}=0.769,95 \% \mathrm{CI}$ : $0.603-0.982 ; P=0.035)$, and propensity score matched analyses $(\mathrm{HR}=0.751,95 \% \mathrm{CI}: 0.590-0.956 ; P=0.020$, Figure 4).

\section{Univariable and Multivariable Analysis of DLBCL Patients}

After PSM, a univariable analysis revealed that low PNI was an adverse factor for the survival of patients $(\mathrm{HR}=0.783$, $P=0.047$, Table 2 ). In addition, age, CNS involvement and IPI appeared to be stronger predictors $(P<0.001)$. However, BCL2, BCL-6, and cell of origin were not independent prognostic factors of DLBCL $(P>0.05)$. Multivariable analysis revealed that PNI remained an independent prognostic factor for DLBCL $(\mathrm{HR}=0.751, P=0.020)$. Compared to patients with low PNI (PNI <44), patients with PNI $\geq 44$ had a high 5-y OS (51.1\% vs $44.8 \%)$.

\section{Prognostic Values of PNI with Clinicopathologic Factors in DLBCL}

Of 564 patients after PSM, 303 cases were positive for BCL-2, and 257 cases were positive for BCL-6. Sixty-four $(11.3 \%)$ patients were with high Ki-67 score $(\geq 0.9)$ and 259 (45.9\%) were non-GCB. PNI in Ann Arbor Stage (III/ $\mathrm{VI})$, ECOG (<2), IPI (LR+LIR), GCB, and BCL-2 negative groups could re-stratify the survival of patients (Figure 5). While PNI in Ann Arbor Stage (I/II), ECOG $(\geq 2)$, IPI (HLR+HR), non-GCB, BCL-2 positive group, BCL-6, Ki-67, and MYC groups could not predict the survival of patients.

Table 2 Univariable and Multivariable Analysis After I:I Ratio PSM

\begin{tabular}{|c|c|c|c|c|c|c|c|}
\hline \multirow[t]{2}{*}{ Variables } & \multicolumn{3}{|c|}{ Univariable Analysis } & \multirow[t]{2}{*}{ Variables } & \multicolumn{3}{|c|}{ Multivariable Analysis } \\
\hline & HR & $95 \% \mathrm{Cl}$ & $\mathbf{P}$ & & HR & $95 \% \mathrm{Cl}$ & $\mathbf{P}$ \\
\hline $\mathrm{PNI}$ & 0.783 & $0.616-0.996$ & 0.047 & PNI & $0.75 I$ & $0.590-0.956$ & 0.02 \\
\hline Age $(y)$ & 1.025 & $1.015-1.036$ & $<0.001$ & Age $(y)$ & 1.019 & $1.008-1.030$ & 0.001 \\
\hline Gender & 0.797 & $0.623-1.020$ & $0.07 \mid$ & CNS involvement & & & \\
\hline CNS involvement & 2.917 & $2.265-3.757$ & $<0.001$ & Absence & & & \\
\hline ECOG & 1.486 & $1.166-1.894$ & 0.001 & Presence & 2.583 & $2.00 \mathrm{I}-3.334$ & $<0.001$ \\
\hline IPI & 2.305 & $1.780-2.984$ & $<0.001$ & IPI & & & \\
\hline Ann Arbor stage & 1.200 & $0.921-1.562$ & 0.177 & $L R+L I R$ & & & \\
\hline PLT & 1.000 & $0.999-1.001$ & 0.777 & $\mathrm{HIR}+\mathrm{HR}$ & 1.967 & $1.512-2.560$ & $<0.001$ \\
\hline
\end{tabular}

Abbreviations: PLT, platelet; CNS involvement, central nervous system involvement. 

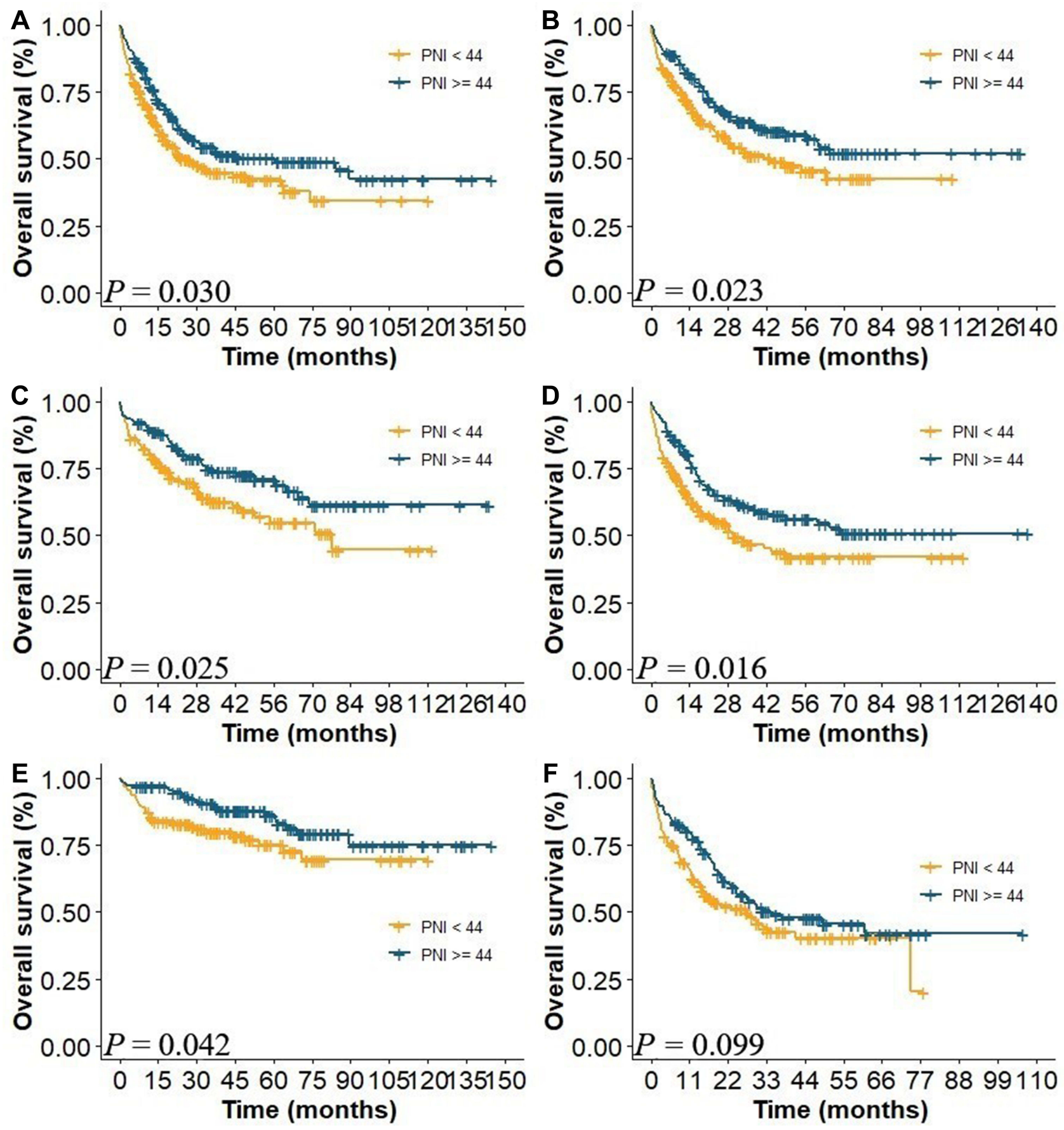

Figure 5 Kaplan-Meier survival curves of DLBCL patients. (A) prognosis of different PNI levels in Ann Arbor stage (III/VI) group; (B) prognosis of different PNI levels in ECOG (<2) group; $(\mathbf{C})$ prognosis of different PNI levels in IPI (LR/LIR) group; (D) prognosis of different PNI levels in GCB group; (E) prognosis of different PNI levels in negative BCL-2 group; (F) prognosis of different PNI levels in positive BCL-6 group.

\section{Discussion}

Diffuse large B-cell lymphoma (DLBCL) is the most common non-Hodgkin's lymphoma with highly heterogeneous. In this retrospective multicenter study, we analyzed the value of PNI on DLBCL. To our knowledge, this is the first study evaluating the prognostic effect of PNI on DLBCL with propensity score matching analysis, and we proved that PNI was an independent factor for patients with DLBCL.

Nutritional status is closely related to the prognosis of lymphoma patients. ${ }^{19-21}$ Nutritional insufficiency may influence poor survival outcomes of DLBCL patients. ${ }^{22}$ Kanemasa et al confirmed that geriatric nutritional risk index could identify a population of poor-risk DLBCL 
patients by NCCN-IPI. ${ }^{23}$ The controlling nutritional status was identified as an independent prognostic factor for OS of DLBCL patients. ${ }^{24,25}$ PNI, a feasible tool for assessing the relationship between immunonutritional status and prognosis of patients has been widely used in acute heart failure, esophageal cancers and lymphoma. ${ }^{10,26-28}$ In this study, we confirmed that PNI was an independent factor for predicting the survival of DLBCL, and we calculated the optimal cut-off point of PNI. According to the maximal chi-square method, 44 was the optimal cut-off point that distinguished between two prognostic groups most effectively, which was close to the results of previous studies. ${ }^{11,12,29,30}$ However, these studies did not take confounding factors into consideration, which might misevaluate the prognostic value on DLBCL. In our study, using propensity score matched analysis, other clinical characteristics of patients were well adjusted, and no differences were confirmed between low PNI and high PNI groups. This reduces the interference of confounders on survival outcome to some extent. Multivariate analysis showed that PNI, age, CNS involvement and IPI were independent prognostic factors for OS. Patients in HIR/HR groups of IPI had poor survival, which was consistent with one of our previous studies. ${ }^{31}$

DLBCL is highly heterogeneous in pathological features and elements of heterogeneity are associated with the prognosis of patients. Therefore, we evaluated the values of PNI on different groups of cell-of-origin, Ann Arbor Stage, ECOG, IPI and other pathological factors. The results confirmed that PNI could not re-stratify BCL-2 positive and non-GCB groups, while PNI could re-stratify BCL-2 negative, Ann Arbor Stage (III/VI) and IPI (LR+LIR) groups. The 5-y OS for malnourished patients was $56.3 \%$ in GCB group and $55.9 \%$ in ECOG $(<2)$ group.

In conclusion, by using propensity score matched analysis, we confirmed that adjusted PNI was an effective prognostic factor for DLBCL patients. However, due to the inherent flaw of the retrospective design, more prospective studies need to be conducted to confirm this result in the future.

\section{Ethics Approval and Informed Consent}

Study approval was obtained from the independent Ethics Committees of each center in HHLWG and met with the Helsinki Declaration. Ethics committees approved the patients or next of kin being contacted by telephone. Written, informed consent was waived, since this study used retrospective data obtained only from hospital medical records.

\section{Acknowledgment}

Thanks to the Huaihai Lymphoma Working Group (HHLWG) for its participation in this study. HHLWG was a non-governmental group established in November 2017 and included 18 medical centers in Huaihai Economic Zone of China. The 18 medical centers are listed as follows:

1. Department of Hematology, Affiliated Hospital of Xuzhou Medical University, Xuzhou, Jiangsu, 221002, China

2. Department of Hematology, Qilu Hospital of Shandong University, Jinan, Shandong, 250012, China

3. Department of Hematology, The Affiliated Hospital of Jining Medical University, Jining, Shandong, 272000, China

4. Department of Hematology, Taian Central Hospital, Taian, Shandong, 271000, China

5. Department of Hematology, The First People's Hospital of Changzhou, Changzhou, Jiangsu, 213003, China

6. Department of Hematology, Yancheng First People's Hospital, Yancheng, Jiangsu, 224001, China

7. Department of Hematology, The First Affiliated Hospital of Anhui Medical University, Hefei, Anhui, 230022, China

8. Department of Hematology, The First People's Hospital of Huaian, Huaian, Jiangsu, 223300, China

9. Department of Hematology, The First People's Hospital of Bozhou, Bozhou, Anhui, 236800, China

10. Department of Hematology, The First People's Hospital of Lianyungang, Lianyungang, Jiangsu, 222002, China

11. Department of Hematology, The First Affiliated Hospital of Zhengzhou University, Zhengzhou, Henan, 450052, China

12. Department of Hematology, The Second Affiliated Hospital of Soochow University, Soochow, Jiangsu, 215000, China

13. Department of Hematology, The People's Hospital of Suqian, Suqian, Jiangsu, 223800, China

14. Department of Hematology, The Henan cancer Hospital, Zhengzhou, Henan, 450008, China

15. Department of Hematology, Affiliated Hospital of Bengbu Medical College, Bengbu, Anhui, 233004, China

16. Department of Hematology, Wanbei Coal Power Group General Hospital, Suzhou, Anhui Province, Suzhou, 234000,China 
17. Department of Hematology, The Affiliated Shuyang Hospital of Xuzhou Medical University/ Shuyang People's Hospital, Suqian, 223600, China

18. Department of Hematology, Fengxian People's Hospital, Xuzhou, 221700, Jiangsu, China

\section{Funding}

This study was funded by Natural Science Foundation of Jiangsu Province, Grant/Award Number: BK20171181; Jiangsu Key Research and Development Project of Social Development, Grant/Award Number: BE2019638; Young Medical Talents of Jiangsu Science and Education Health Project, Grant/Award Number: QNRC2016791.

\section{Disclosure}

The authors declare no conflicts of interest.

\section{References}

1. Shipp MA. International Non-Hodgkin's Lymphoma Prognostic Factors Project. A predictive model for aggressive non-Hodgkin's lymphoma. $N$ Engl J Med. 1993;329(14):987-994. doi:10.1056/ NEJM199309303291402

2. Zhou Z, Sehn LH, Rademaker AW, et al. An enhanced International Prognostic Index (NCCN-IPI) for patients with diffuse large B-cell lymphoma treated in the rituximab era. Blood. 2014;123(6):837-842. doi:10.1182/blood-2013-09-524108

3. Tisdale MJ. Cachexia in cancer patients. Nat Rev Cancer. 2002;2 (11):862-871. doi:10.1038/nrc927

4. Gupta D, Lis CG, Granick J, Grutsch JF, Vashi PG, Lammersfeld CA. Malnutrition was associated with poor quality of life in colorectal cancer: a retrospective analysis. $J$ Clin Epidemiol. 2006;59 (7):704-709. doi:10.1016/j.jclinepi.2005.08.020

5. Yang L, Xia L, Wang Y, et al. Low prognostic nutritional index (PNI) predicts unfavorable distant metastasis-free survival in nasopharyngeal carcinoma: a propensity score-matched analysis. PLoS One. 2016;11(7):e0158853. doi:10.1371/journal.pone.0158853

6. Lien YC, Hsieh CC, Wu YC, et al. Preoperative serum albumin level is a prognostic indicator for adenocarcinoma of the gastric cardia $J \quad$ Gastrointest Surg. 2004;8(8):1041-1048. doi:10.1016/j. gassur.2004.09.033

7. Buzby GP, Mullen JL, Matthews DC, Hobbs CL, Rosato EF. Prognostic nutritional index in gastrointestinal surgery. Am J Surg. 1980;139(1):160-167. doi:10.1016/0002-9610(80)90246-9

8. Fanetti G, Polesel J, Fratta E, et al. Prognostic nutritional index predicts toxicity in head and neck cancer patients treated with definitive radiotherapy in association with chemotherapy. Nutrients. 2021;13(4):1277. doi:10.3390/nu13041277

9. Liu N, Jiang A, Zheng X, et al. Prognostic nutritional index identifies risk of early progression and survival outcomes in advanced non-small cell lung cancer patients treated with PD-1 inhibitors. J Cancer. 2021;12(10):2960-2967. doi:10.7150/jca.55936

10. Yao N, Hou Q, Zhang S, et al. Prognostic nutritional index, another prognostic factor for extranodal natural killer/T cell lymphoma, nasal type. Front Oncol. 2020;10:877. doi:10.3389/fonc.2020.00877

11. Go SI, Park S, Kang MH, Kim HG, Kim HR, Lee GW. Clinical impact of prognostic nutritional index in diffuse large B cell lymphoma. Ann Hematol. 2019;98(2):401-411. doi:10.1007/s00277018-3540-1
12. Yu W, Guo Q, Wang Z, et al. Clinical significance of prognostic nutritional index for patients with diffuse large B-cell lymphoma. Nutr Cancer. 2019;71(4):569-574. doi:10.1080/01635581.2018.1540718

13. Zhou Q, Wei Y, Huang F, et al. Low prognostic nutritional index predicts poor outcome in diffuse large B-cell lymphoma treated with R-CHOP. Int J Hematol. 2016;104(4):485-490. doi:10.1007/s12185016-2052-9

14. Kim DH, Pieper CF, Ahmed A, Colon-Emeric CS. Use and interpretation of propensity scores in aging research: a guide for clinical researchers. J Am Geriatr Soc. 2016;64(10):2065-2073. doi:10.1111/ jgs. 14253

15. Reiffel JA. Propensity-score matching: optimal, adequate, or incomplete? J Atr Fibrillation. 2018;11(4):2130. doi:10.4022/ jafib. 2130

16. Su CW, Fang KC, Lee RC, et al. Association between esophagogastric varices in hepatocellular carcinoma and poor prognosis after transarterial chemoembolization: a propensity score matching analysis. J Formos Med Assoc. 2020;119(2):610-620. doi:10.1016/j. jfma.2019.09.003

17. Baek S, Park SH, Won E, Park YR, Kim HJ. Propensity score matching: a conceptual review for radiology researchers. Korean J Radiol. 2015;16(2):286-296. doi:10.3348/kjr.2015.16.2.286

18. He C, Wang W, Chen Q, et al. Factors associated with stroke among patients with type 2 diabetes mellitus in China: a propensity score matched study. Acta Diabetol. 2021;58(11):1513-1523. doi:10.1007/ s00592-021-01758-y

19. Paccagnella A, Morassutti I, Rosti G. Nutritional intervention for improving treatment tolerance in cancer patients. Curr Opin Oncol. 2011;23(4):322-330. doi:10.1097/CCO.0b013e3283479c66

20. Ravasco P. Nutrition in cancer patients. J Clin Med. 2019;8(8):1211. doi: $10.3390 / \mathrm{jcm} 8081211$

21. Mantzorou M, Koutelidakis A, Theocharis S, Giaginis C. Clinical value of nutritional status in cancer: what is its impact and how it affects disease progression and prognosis? Nutr Cancer. 2017;69 (8):1151-1176. doi:10.1080/01635581.2017.1367947

22. Park S, Han B, Cho JW, et al. Effect of nutritional status on survival outcome of diffuse large B-cell lymphoma patients treated with rituximab-CHOP. Nutr Cancer. 2014;66(2):225-233. doi:10.1080/ 01635581.2014 .867065

23. Kanemasa Y, Shimoyama T, Sasaki Y, Hishima T, Omuro Y. Geriatric nutritional risk index as a prognostic factor in patients with diffuse large B cell lymphoma. Ann Hematol. 2018;97(6):999-1007. doi:10.1007/s00277-018-3273-1

24. Nagata A, Kanemasa Y, Sasaki Y, et al. Clinical impact of controlling nutritional status score on the prognosis of patients with diffuse large B-cell lymphoma. Hematol Oncol. 2020;38(3):309-317. doi:10.1002/ hon. 2732

25. Baysal M, Bas V, Demirci U, et al. The utility of CONUT score in diffuse large B cell lymphoma patients. Niger J Clin Pract. 2021;24 (8):1194-1199.

26. Cheng YL, Sung SH, Cheng HM, et al. Prognostic nutritional index and the risk of mortality in patients with acute heart failure. $J \mathrm{Am}$ Heart Assoc. 2017;6(6). doi:10.1161/JAHA.116.004876

27. Okadome K, Baba Y, Yagi T, et al. Prognostic nutritional index, tumor-infiltrating lymphocytes, and prognosis in patients with esophageal cancer. Ann Surg. 2020;271(4):693-700. doi:10.1097/ SLA.0000000000002985

28. Paydas S, Lacin S, Dogan M, et al. Easier and more explanatory indices by integrating leukocyte lymphocyte ratio (LLR) and prognostic nutritional index (PNI) to IPS systems in cases with classical Hodgkin lymphoma. Leuk Res. 2021;107:106586. doi:10.1016/j. leukres.2021.106586

29. He J, Yin H, Xia Y, et al. Prognostic nutritional index, a novel biomarker which predicts worse prognosis in diffuse large B cell lymphoma. Leuk Res. 2021;110:106664. doi:10.1016/j. leukres.2021.106664 
30. Perisa V, Zibar L, Knezovic A, Perisa I, Sincic-Petricevic J, Aurer I. Prognostic nutritional index as a predictor of prognosis in patients with diffuse large B cell lymphoma. Wien Klin Wochenschr. 2017;129 (11-12):411-419. doi:10.1007/s00508-016-1077-7
31. Sang W, Zhou H, Qin Y, et al. Risk stratification model based on VEGF and International Prognostic Index accurately identifies low-risk diffuse large B-cell lymphoma patients in the rituximab era. Int $J$ Hematol. 2021;114(2):189-198. doi:10.1007/s12185-021-03145-3

\section{Publish your work in this journal}

The Journal of Inflammation Research is an international, peerreviewed open-access journal that welcomes laboratory and clinical findings on the molecular basis, cell biology and pharmacology of inflammation including original research, reviews, symposium reports, hypothesis formation and commentaries on: acute/chronic inflammation; mediators of inflammation; cellular processes; molecular mechanisms; pharmacology and novel anti-inflammatory drugs; clinical conditions involving inflammation. The manuscript management system is completely online and includes a very quick and fair peerreview system. Visit http://www.dovepress.com/testimonials.php to read real quotes from published authors. 